Author(s): Brack, KE (Brack, K. E.); Watkins, N (Watkins, N.); Pyner, S (Pyner, S.); Coote, JH (Coote, J. H.) Title: A physiological role for nitric oxide in the centrally mediated sympathetic and somatomotor ejaculatory response in anesthetized male Wistar rats Source: NEUROSCIENCE, 150 (2): 487-497 DEC 52007 


\section{A physiological role for NO in the centrally mediated sympathetic and somatomotor ejaculatory response in anaesthetised male Wistar rats}

Brack KE $\mathrm{K}^{\mathrm{a}}$, Watkins $\mathrm{N}^{\mathrm{b}}$, Pyner $\mathrm{S}^{\mathrm{b}}$ and Coote $\mathrm{JH}^{\mathrm{c}} \dagger$

${ }^{\mathrm{a}}$ Dept. of Cardiovascular Sciences, University of Leicester, UK, ${ }^{\mathrm{b}}$ School of Biological and Biomedical Sciences, University of Durham, UK, ${ }^{\mathrm{c} D e p t . ~ o f ~ P h a r m a c o l o g y, ~ D i v i s i o n ~}$ of Neuroscience, University of Birmingham, UK,

$\dagger$ Address for correspondence:

Professor John H Coote

Department of Pharmacology

Division of Neuroscience

Medical School

University of Birmingham

Vincent Drive

Edgbaston

Birmingham

B15 2TT

UK

Tel: +44 (0) 1214146916

Fax: +44 (0) 1214146919

E-mail: j.h.coote@bham.ac.uk

Section Editor: M. Herkenham (Systems Neuroscience) 


\section{Abbreviations}

$\mathrm{BS}$; bulbospongiosus muscle

BP; mean arterial blood pressure

EMG; electromyographic

HR: heart rate

L-Arg; L-Arginine

L-NAME; $\mathrm{N}^{(\mathrm{G})}$ nitro-L-Arginine methyl ester

NOS; nitric oxide synthase

PCA; p-Chloroamphetamine

PB; phosphate buffer

PBS; phosphate buffer saline

TRIM; 1-(2-trifluoro-methylphenyl) imidazole

VDN; vas deferens nerve 


\begin{abstract}
Neurones in the lumbosacral spinal cord are known to play a significant role in ejaculation. In these same areas neurones containing nitric oxide synthase (NOS) have been described. This raised the question as to whether there is a physiological role for nitric oxide in the spinal cord in sexual behaviour. We first established immunohistochemical localisation of NOS positive neurones in the lumbosacral spinal cord. NOS positive neurones were found in several areas of the lumbosacral cord. Namely the intermediolateral column (IML) at $\mathrm{L}_{1}-\mathrm{L}_{4}$ and sacral cord; the dorsal gray commissure above the central canal at $\mathrm{L}_{1}-\mathrm{L}_{2}$; the ventral gray area of lamina $\mathrm{X}$ below the central canal at $\mathrm{L}_{3}-\mathrm{L}_{4}$; the superficial laminae of the dorsal horn at all levels. Secondly, we examined the role of $\mathrm{NO}$ in the generation of synchronised bursting activity within the vas deferens nerve and associated penile muscles, typical of sexual responses in the male anaesthetised rat. NO modulators were applied directly to the spinal cord at $\mathrm{T}_{13}-\mathrm{L}_{4}$ via a catheter placed subdurally (intrathecal) and their effect on the genital responses evoked by systemic administration of p-Chloroamphetamine (PCA) or apomorphine (apo) (both $1 \mathrm{mg} / \mathrm{kg})$ observed. All responses evoked by PCA $(\mathrm{n}=4)$ or apo $(\mathrm{n}=3)$ were abolished or reduced ( $\mathrm{n}=1$ ) during intrathecal NOS inhibition using $\mathrm{N}^{(\mathrm{G})}$ nitro-L-Arginine methyl ester (L-NAME, 200mM, 20- $\mu \mathrm{l}$ ). NOS inhibition using L-NAME was reversed with simultaneous intrathecal application of the NO substrate L-Arginine (100mM, 20- $\mu 1$, $\mathrm{n}=3$ ). The selective neuronal NOS inhibitor 1-(2-trifluoro-methylphenyl) imidazole (100mM, 20- $\mu$ l, TRIM) also abolished all responses evoked by PCA $(n=3)$. There was evidence that the responses within the VDN after PCA or apo were enhanced following NO donation using sodium nitroprusside (SNP, $1 \mathrm{mM}, 20-\mu \mathrm{l})$. Furthermore, a PCA-like response within the VDN was evoked following intrathecally applied L-Glutamic acid $(200 \mathrm{mM}, 20-\mu \mathrm{l})$ in 6 out of 26 animals and also by intrathecal SNP in 2 out of 8 animals. In conclusion the results suggest a significant excitatory role for NO in the bursting pattern of synchronised discharge generated in autonomic and somatic outflows from the lumbosacral cord by neurones governing ejaculation.
\end{abstract}

Key words: vas deferens, ejaculation, sexual climax, nitric oxide, spinal pattern generator, intrathecal, NOS immunohistochemistry 


\section{Introduction}

Several immunohistochemical studies have shown that spinal sympathetic nuclei contain a high density of nitric oxide (NO) synthesising enzymes (NOS) or the co-factor nicotinamide adenine dinucleotide phosphate diaphorase (Anderson, 1992; Anderson et al., 1995; Blottner and Baumgarten, 1992; Valtschanoff et al., 1992; Dunn et al., 1993; Saito et al., 1994). Physiological studies have shown that NO acts as a messenger molecule in spinal sympathetic networks where it may enhance post synaptic potentials via an action on the presynaptic transmitter release (Wu and Dun, 1995), or inhibit sympathetic neurones retrogradely via glycine interneurones (Wu and Dun, 1996; Yang et al., 2004). The actions appear to participate in sympathetic vasomotor regulation (Hakim et al., 1995; Lee at al., 1996; Garcia et al., 1997, Yang et al., 2004). However the distribution of NOS positive spinal autonomic neurones is extensive suggesting that NO may have important actions on other autonomic functions. Conspicuous groups of NOS immunoreactive neurones are found in the principal autonomic nuclei of the upper lumbar and well as the lower lumbar/sacral segments (Dun et al., 1993). This may indicate that NO plays a role in genital functions.

We have recently described a highly repeatable bursting pattern of intense sympathetic activity in a vas deferens nerve (VDN) that is associated with contraction of the vas deferens, emission and other ejaculatory markers evoked by i.v. administration of pChloroamphetamine (PCA) (Stafford et al., 2006a) or apomorphine (Stafford and Coote 2006). These PCA evoked responses are partly mediated by 5- $\mathrm{HT}_{2 \mathrm{C}}$ receptors on neurones in the lumbosacral spinal cord (Stafford et al., 2006b). Such activation will result in an increase in intracellular calcium concentration, which in NOS positive neurones could release NO with a subsequent enhancement of excitatory synaptic potentials (Wu and Dun, 1995). Since lumbar sympathetic neurones contain NOS (Dun et al., 1993) it is possible that VDN neurones may also be NOS positive, possibly providing a role for $\mathrm{NO}$ in the bursting pattern of sympathetic activity induced by i.v. PCA or apomorphine. NOS immunoreactivity in neurones of the lower lumbar and sacral spinal cord has also been described (Saito et al., 1994) although no attempt has been made to relate their location to that of neurones involved in ejaculation. Therefore the present study first sought to confirm the distribution and location of NOS positive neurones in the 
lumbosacral cord and to correlate this with regions considered to play a role in sexual responses. Secondly, using the anaesthetised rat model first described by Stafford et al (2006a) we determined whether NO in the lumbosacral cord participated physiologically in the generation of the spinal ejaculatory pattern of sympathetic and penile muscle response.

\section{Experimental procedures}

All experiments were approved by the local ethics committee of the University of Birmingham and were performed under a Home Office licence in accordance with the UK Animal (Scientific Procedures) Act 1986. All procedures were conducted on deeply anaesthetised animals that minimised suffering and all efforts were made to minimise the number of animals used. Animals were killed with an overdose of Urethane at the termination of the experiment.

\section{Surgical preparation}

32 male Wistar rats, weighing $281.3 \pm 4.8 \mathrm{~g}$, were used. Anaesthesia was induced using $5 \%$ isoflurane. The right femoral vein was cannulated using $0.96 \mathrm{~mm}$ (O.D.) polyethylene tubing and animals were transferred onto urethane anaesthesia $(1.53 \pm 0.03 \mathrm{~g} / \mathrm{kg}$ i.v. $)$. The depth of anaesthesia was checked at regular intervals using the appearance and briskness of cornea/pedal reflexes and supplementary injections of $0.1 \mathrm{ml}$ urethane were used if necessary. The right femoral artery was cannulated using $0.96 \mathrm{~mm}$ (O.D.) polyethylene tubing (Fisher Scientific, Loughborough, UK) filled with heparinised saline (20 IU/ml). The arterial cannula was connected to a pressure transducer (Capto SP 844, ADInstruments, Oxfordshire, UK), Bridge Amplifier (Model ML221, ADInstruments) and Powerlab system (ADInstruments) to monitor and record blood pressure and heart rate using Chart v4.1.1 (ADInstruments). The trachea was intubated using a truncated 6F (yellow) luer cannula (Portex Ltd, Kent, UK) to maintain a clear airway and record airflow and rate of respiration via a spirometer (Model ML141, ADInstruments).

\section{Intrathecal cannulation}

The spinal subarachnoid space was cannulated for the intrathecal (i.t.) administration of drugs. For this the rat was placed in the prone position and the skin overlaying the lumbar and sacral vertebrae was shaved and the region of the $T_{13} / L_{1}$ indicated using an indelible marker. An incision was made to expose the lumbar spine and the dorsal surface of the $\mathrm{L}_{5}$ 
vertebra exposed after removal of the overlaying muscle. The dorsal lamina of the $\mathrm{L}_{5}$ vertebra was partially removed and a PE-10 polyethylene catheter (Storkson et al, 1996), connected to a syringe filled with artificial cerebrospinal fluid (ASCF), was inserted under the dura through a small incision and advanced rostrally to the $\mathrm{L}_{1}-\mathrm{L}_{2}$ region. Care was taken to prevent damage to the nerve roots and spinal cord. The catheter was held in place by a ligature in the lumbar muscle and the wound was closed with sutures. Tip position was confirmed at necropsy. ACSF was made fresh daily and had the following composition (in mM): $\mathrm{NaCL}, 120.0$; $\mathrm{KCL}, 5.0 ; \mathrm{MgCL}_{2}, 2.0$; $\mathrm{CaCL}_{2}$, 5.0; Glucose, 25.0; HEPES, 10.0, pH 7.4.

\section{VDN exposure}

The animal was placed and remained in the supine position for the duration of the experiment. Surgery was then performed to access the VDN for recording as described fully by Stafford et al (2006a). Briefly, a ventral midline incision was made from the base of the sternum as far as the corpus penis, to access the abdomen and viscera. The animal was subsequently placed on a heating blanket (Harvard Instruments) and temperature monitored using a rectal probe, maintaining temperature at $36^{\circ} \mathrm{C}$.

The abdomen was held open by securing the abdominal wall to a metal oval frame. The colon, ileum, seminal vesicles and bladder were secured aside in order to clearly access the nerve to the vas deferens. The nerve was dissected away from the surrounding tissue and mounted onto custom made $0.35 \mathrm{~mm}$ silver bipolar hook electrodes and the abdominal cavity was filled with liquid paraffin (VWR, Lutterworth, UK) maintained at body temperature and to provide electrical isolation and prevent VDN damage from dehydration.

\section{Recording VDN activity}

VDN activity was amplified using a Neurolog amplifier (module NL104, Digitimer Ltd, Welwyn Garden City, UK), filtered at $50 \mathrm{~Hz}$ (low frequency) to $3 \mathrm{kHz}$ (high frequency) (module NL125) and displayed on an oscilloscope (Gould, Ilford, UK). The signal was recorded simultaneously along with the cardiovascular and respiratory parameters on a Power Mac G4 personal computer using a Powerlab 8SP system and Chart acquisition software (v4.1.1, ADInstruments). VDN activity was converted online into firing frequency using a window discriminator within the Chart software set to remove 
electrical noise, which was confirmed by crushing the nerve at the end of the experiment. Animals were left to recover for at least one hour before commencing with drug administrations.

\section{Recording of bulbospongiosus muscle activity}

A 2-3 cm midline longitudinal incision was made in the scrotum and the bulbospongiosus muscle exposed at the base of the penile bulb. Two silver wire electrodes were placed in the bulbospongiosus muscle and electromyographic (EMG) activity amplified (Neurolog, module NL104) and filtered at $50 \mathrm{~Hz}$ (low frequency) to $3 \mathrm{kHz}$ (high frequency) (module NL125) and recorded along with VDN activity.

\section{Experimental protocols}

\section{Apomorphine and PCA tests}

Apomorphine or PCA were administered intravenously (in $0.9 \%$ saline) at a dose of $1.0 \mathrm{mg} / \mathrm{kg}$ (Stafford et al., 2006a; Stafford and Coote 2006) via the femoral vein and washed in with $0.1 \mathrm{ml}$ saline. Each injection was carried out over a 5-second period and successive administrations were performed at hourly intervals.

\section{i.t. administration of drugs}

Initial confirmation of the location of the catheter tip was established by i.t. administration of L-Glutamic acid. All animals used in this study demonstrated a clear increase in VDN activity after L-Glutamic acid administration and therefore the position of the i.t. catheter was considered suitable to investigate the role of spinal cord NO in the ejaculatory response. As a control, $20-\mu 1$ of ACSF was tested.

Modification by i.t. drugs of the response to apomorphine was examined between i.v. administrations 1 and 2 of this agent whereas the effect of i.t. drugs on PCA was examined between i.v. administrations 2 and 3 of this agent. Unless otherwise specified, i.t. injections took place 5 minutes prior to i.v. administration of apomorphine or PCA. Each drug was tested in separate animals. Drugs were administered in a volume of $20 \mu 1$ at the rate of $0.5 \mu \mathrm{l} / \mathrm{s}$. This procedure ensured that the $T_{13}$ to $L_{4}$ segments of spinal cord were reached by the drugs (confirmed by i.t. $20 \mu \mathrm{l}$ of a solution containing pontamine sky blue) and without significant pressure change (confirmed by the absence of responses following i.t. $20 \mu \mathrm{ACSF}$ ). 


\section{Experimental groups}

Experiments were divided into the following groups:

1) Characteristics of response to i.v. PCA $(n=26)$ or apomorphine $(n=6)$.

2) Reproducibility of VDN response between sequential administrations of PCA $(n=5)$.

3) Inhibition of NO production with the intrathecal application of the non-specific NOS inhibitor $\mathrm{N}^{(\mathrm{G})}$ nitro-L-Arginine methyl ester (L-NAME, $\mathrm{n}=7$ ) and neuronal specific NOS inhibitor 1-(2-trifluoro-methylphenyl) imidazole (TRIM, n=3).

4) Reversal of the effect of L-NAME with the simultaneous intrathecal application of the NO precursor L-Arginine (L-NAME + L-Arg, n=3).

5) Effect of Intrathecal administration of L-Arginine on the response within the VDN using i.v. PCA (L-Arg, $n=5)$.

6) Intrathecal NO donation with the NO donor sodium nitroprusside ( $\mathrm{SNP}, \mathrm{n}=8$ ).

\section{Data analysis}

\section{Blood pressure, heart rate and ventilation}

Mean arterial blood pressure (BP), heart rate (HR) derived from the blood pressure record and the rate of respiration determined from airflow movements were analysed at baseline (BL) and during the peak level achieved after i.v. apomorphine and PCA administration. The temporal changes in each parameter were also recorded, namely the latency to and duration of the haemodynamic, chronotropic and ventilatory response elicited by i.v. apomorphine or PCA.

\section{VDN activity}

A 'response' is defined as one or more synchronous oscillatory bursts in VDN activity. The characteristics of the patterned responses elicited by i.v. PCA or apomorphine have been described previously (Stafford and Coote, 2006; Stafford et al., 2006a). Parameters measured were as follows: the latency to the first response; overall duration of all responses from the time of PCA or apomorphine administration to the end of the last response; the total number of responses; the mean duration of all individual response(s); the number of synchronous oscillatory bursts that occur within a response(s); the frequency of synchronous oscillatory bursts that occur within a response(s); the baseline firing rate prior to PCA or apomorphine administration; the firing rate immediately prior 
to the synchronous oscillatory bursts, the maximum firing rate of the synchronous oscillatory bursts within the response(s).

All data are presented as mean \pm S.E.M. Statistical analysis was carried out using one-way ANOVA and paired students t-test where appropriate using GraphPad Prism software (v4, GraphPad Software, San Diego, US). P $<0.05$ was considered significant.

\section{Immunohistochemical identification of NOS positive neurones in the region of the ejaculatory pattern generator}

To obtain tissue for immunhistochemical identification of NOS (NOS-IR) within the spinal cord, a separate group of 3 rats were killed and retrogradely perfused via the descending aorta with $400 \mathrm{ml}$ heparinised saline $(10 \mathrm{IU} / \mathrm{ml})$ followed by $400 \mathrm{ml} 4 \%$ paraformaldehyde with $0.25 \%$ glutaraldehyde in $0.1 \mathrm{M}$ phosphate buffer $(\mathrm{PB})(\mathrm{pH} 7.4)$. Each spinal cord was post fixed for 2 hours and then transferred to $30 \%$ sucrose in $0.1 \mathrm{M}$ $\mathrm{PB}$ at $4^{\circ} \mathrm{C}$ overnight to afford cryoprotection. $40-50 \mu \mathrm{m}$ transverse or longitudinal sections were cut using a freezing mictrotome. After an initial rinse in PBS for 10 minutes with gentle agitation, sections were blocked (PBS containing 10\% normal goat serum [Vector Labs, Burlingame, US] and 0.1\% Triton-X 100 [Sigma-Aldrich, Poole, UK]) for 30 minutes. After 3 (x10 mins) washes in PBS, sections were incubated in primary antibody for neuronal NOS (1:200 dilution [Sc648, Santa Cruz Biotechnology, California, US] along with 1\% normal goat serum and 0.1\% Triton-X 100 in PBS) for 24 hours at $4^{\circ} \mathrm{C}$. Sections were subsequently washed in PBS ( $\left.3 \times 10 \mathrm{mins}\right)$ and incubated in secondary antibody (1:500 [BA-1000, Vector labs] together with 1\% normal goat serum and $0.1 \%$ Triton-X 100) for 1 hour. After further washes ( 3 x $10 \mathrm{~min}$ in PBS) the reaction product was visualised after incubation in ABC (kit PK-6100, Vector Labs, 30 minutes, followed by 2 x 10 minutes washes in PBS) using a VIP kit (SK-4600, Vector Labs, Peterborough, UK). Following further washes, sections were mounted onto gelatinised slides, dried in air overnight and then dehydrated through ascending series of alcohols, cleared in Histoclear (National Diagnostics, Atlanta, US) and mounted using Histomount (National Dioagnostics, Atlanta, US).

\section{Specificity of NOS immunoreactivity}

The nNOS antisera were an affinity purified rabbit polyclonal antibody against rat brain nNOS (Santa Cruz Biotechnology Inc.). This NOS antibody has been extensively 
characterised and shown to be specific for isoform 1 of rat brain NOS (nNOS). The present study followed the protocol and used the same antibody as described by Zheng et al (2006). Controls for false positive results were carried out on alternate spinal cord sections incubated in primary antibody (0.1M PBS, $0.1 \%$ Triton-X 100, $1 \%$ normal goat serum) followed by a further similar incubation with no secondary antibody. A similar procedure was carried out on a third group of sections from the same regions of spinal cord but without primary antibody for the final incubation but with secondary antibody included for the second incubation. All sections were then incubated in $\mathrm{ABC}$ solution using a VIP kit (SK-4600, Vector labs) processed and visualised as described above for the test solutions. There was a complete absence of nNOS immunoreactivity in these control section.

\section{Drugs}

Apomorphine (Tocris, Bristol, UK) and PCA (Sigma-Aldrich, Dorset, UK) were dissolved in physiological saline and administered i.v. at a dose of $1 \mathrm{mg} / \mathrm{kg}$, washed in with $0.1 \mathrm{ml}$ saline. All drugs administered i.t. were purchased from Sigma-Aldrich and dissolved in artificial ACSF at the following concentrations: L-Glutamic acid (L-Glu, $200 \mathrm{mM}$ ); $\mathrm{N}^{(\mathrm{G})}$ nitro-L-arginine methyl ester (L-NAME, 200mM); L-Arginine (L-Arg, 100mM); 1-(2-trifluoro-methylphenyl) imidazole (TRIM, 150mM); Sodium nitroprusside (SNP, 1mM). The concentrations used were based on Yang et al (2004), Stafford and Coote (2006) and Stafford et al (2006a).

\section{Results}

\section{NOS-IR in the lumbosacral cord}

NOS positive neurones were clearly identified in both transverse and longitudinal sections of the thoracic and lumbosacral cord (Fig1, Fig 2, Fig 3). These were most conspicuous in four areas. 1) The intermediolateral column (IML) at thoracic levels (Fig 1A, B; Fig 2A, B, Fig 3) at $\mathrm{L}_{1}-\mathrm{L}_{4}$ (Fig 1C, D; Fig 2C, D, Fig 3) and less dense at $\mathrm{L}_{6}-\mathrm{S}_{4}$ (Fig 1E, Fig 3). 2) A group of neurones in the dorsal gray commissure above the central canal at thoracic levels (Fig 1A, B, Fig 3) but most conspicuous at $\mathrm{L}_{1}-\mathrm{L}_{2}$ (Fig 1C, Fig 3) which corresponds to the central autonomic area (CA). 3) A group of neurones lying either side of the central canal and extending ventrally in the gray matter of lamina $\mathrm{X}$ to lie below the central canal most prominently at $\mathrm{L}_{3}-\mathrm{L}_{4}$ (Fig 1D; Fig 2C, D, Fig 3). 4) The 
superficial laminae of the dorsal horn at all levels. There was a distinct absence of labelling in neurones of the ventral horn (Fig 3) at all levels.

Insert figures 1, 2 and 3 near this position

\section{Actions of intravenous PCA}

\section{Blood pressure, heart rate and ventilation}

Immediately following PCA ( $1 \mathrm{mg} / \mathrm{kg}$, i.v. $\mathrm{n}=26)$ there was a small increase in BP (mean $16.0 \pm 1.6 \%, \mathrm{P}<0.05$ ) from a baseline of $98.4 \pm 3.0 \mathrm{mmHg}$ to $113.7 \pm 3.0 \mathrm{mmHg}$ after which it slowly fell to a lower level than control $(89.3 \pm 3.2 \mathrm{mmHg})$ where it remained for 10 mins before recovering towards control levels. Heart rate typically increased (mean $12.7 \pm 2.1 \%$ ) from a baseline of $452.2 \pm 7.2 \mathrm{bpm}$ but this failed to reach significance $(\mathrm{P}>0.05)$. Respiration also showed a non-significant increase from a baseline of $133.1 \pm 4.1$ breaths per minute to $142.5 \pm 6.9$ breaths per minute $(\mathrm{P}>0.05,14.4 \pm 3.1 \%)$ before slowly recovering to $120.0 \pm 4.5$ breaths per minute.

\section{VDN activity}

In 26 rats, PCA ( $1 \mathrm{mg} / \mathrm{kg}$, i.v.) initially caused a progressive increase in VDN firing rate to a mean of $76.1 \pm 5.9 \mathrm{~Hz}$ from a baseline of $31.0 \pm 1.9$. This was followed by a series of intense bursts of nerve activity (peak firing frequency of 151.6 $\pm 6.5 \mathrm{~Hz}$ ). Each sequence comprised of a mean of $6.4 \pm 0.6$ bursts of synchronised discharge that we have previously defined as a response (Stafford et al, 2006a). An example from one rat is shown in figure 4. The duration of each individual PCA response was $17.1 \pm 1.9 \mathrm{~s}$ and the repetitive rate of each burst in a response had a mean of $0.388 \pm 0.012 \mathrm{~Hz}$. The first response occurred after a latency of $174.6 \pm 22.8 \mathrm{~s}$ and the mean number of responses to a single dose of PCA was 2.9 \pm 0.4. The whole sequence of responses was completed within $632.5 \pm 124.4 \mathrm{~s}$.

Insert figure 4 near this position

We observed that each period of rhythmic bursting activity in the VDN was accompanied by simultaneous rhythmic contractions of the penile muscles as indicated by bursts of activity in the BSm EMG activity trace (Fig 5) and emission of seminal fluid from the glans penis but there was no obvious evidence of engorgement of the glans although retraction of the skin around the glans occasionally occurred (Stafford et al, 2006a). The ejaculatory events occurred well after the cardio-respiratory changes at a time when the latter were stable and virtually back to control values. 


\section{Action of intravenous apomorphine}

\section{Blood pressure, heart rate and ventilation}

Apomorphine $(1 \mathrm{mg} / \mathrm{kg})$ elicited a profound decrease in BP with a mean change in 6 rats of $42.2 \pm 7.0 \%(\mathrm{P}<0.001)$ from a mean of $99.2 \pm 8.9 \mathrm{mmHg}$ to a mean of $57.0 \pm 2.8 \mathrm{mmHg}$. This was accompanied by a decrease in heart rate of $23.6 \pm 6.4 \%$ (from $481.2 \pm 14.7 \mathrm{bpm}$ to $457.6 \pm 16.3 \mathrm{bpm}, \mathrm{P}>0.05)$. These changes occurred almost immediately after apomorphine administration but thereafter BP and heart rate slowly returned towards control over 5 mins. Ventilation was not recorded in these animals.

\section{VDN activity}

In 6 rats, apomorphine $(1 \mathrm{mg} / \mathrm{kg}$ i.v. $)$ initially caused a progressive increase in VDN firing rate to a mean of $59.4 \pm 13.1 \mathrm{~Hz}$ from a baseline of $40.1 \pm 14.4 \mathrm{~Hz}$. This was followed by a series of intense bursts of activity (mean peak frequency $145.1 \pm 16.3 \mathrm{~Hz}$.) each sequence comprising $4.9 \pm 0.3$ bursts. The duration of each response was $9.8 \pm 0.6 \mathrm{~s}$ and the repetition rate of each burst in a response was $0.496 \pm 0.008 \mathrm{~Hz}$. The first response occurred after a latency of $192.1 \pm 83.6 \mathrm{~s}$ and the mean number of responses to a single dose of apomorphine was $6.7 \pm 1.5$. The whole sequence of responses was completed within $1509.8 \pm 365.4 \mathrm{~s}$. None of the changes in VDN activity was correlated with cardiorespiratory events.

Like for PCA, the periods of rhythmic bursting were always accompanied by simultaneous rhythmic contractions of the penile muscles and emission of seminal fluid from the glans penis.

\section{Effect of sequential dosing of PCA on VDN activity}

In 6 rats we examined how well the VDN responses were reproduced to repeated doses of i.v. PCA. All the rats were implanted with an intrathecal catheter to ensure the preparations were comparable to the main series of rats where drugs were also applied to the spinal cord. In only 2 rats of this series was there a response to the first dose of PCA. This was confirmed in the 26 rats subjected to testing with PCA i.v. and intrathecally applied drugs, where less than $20 \%$ showed a response to the first dose of PCA. In the 6 control rats as well as in the 26 'test' rats all animals responded to the second administration. All 6 control rats responded to subsequent administrations of PCA i.v., that is the third, fourth and even the fifth dose. For experimental purposes we therefore 
adopted a protocol to test effects of intrathecal drugs between administration 2 and 3 . Table 1 shows the characteristics of the PCA responses in VDN for repetition doses 2 and 3. Although there were differences, in such parameters as number of responses, latency to the first response and overall duration of the sequence of responses, most parameters were quite similar and none of the differences were statistically significant.

Insert table 1 near this position

\section{Effect of ACSF and L-Glutamic acid i.t.}

Control intrathecal injections of $20-\mu l$ artificial cerebrospinal fluid $(n=26)$ did not significantly $(\mathrm{P}>0.05)$ alter blood pressure $(92.6 \pm 3.8 \mathrm{mmHg}$ to $91.8 \pm 4.1 \mathrm{mmHg})$, heart rate $(457.8 \pm 7.2$ to $458.6 \pm 7.2 \mathrm{bpm})$, rate of respiration (141.4 \pm 4.4 breaths per minute to $142.7 \pm 4.2$ breaths per minute) or mean firing rate within the VDN $(41.2 \pm 5.0 \mathrm{~Hz}$ to $40.6 \pm 4.5 \mathrm{~Hz})$.

To check that the i.t. catheter was located at $\mathrm{L}_{1}-\mathrm{L}_{2}$ level we injected L-Glutamic acid $(200 \mathrm{mM}, 20-\mu \mathrm{l})$. This elicited a significant increase in basal firing rate of VDN activity from a mean of $40.2 \pm 4.4 \mathrm{~Hz}$ to $56.6 \pm 4.4 \mathrm{~Hz}(\mathrm{P}<0.001)$. Remarkably in 6 of the 26 rats the initial increase in VDN activity was followed by a single PCA-like response. This VDN response occurred after a latency of $26.5 \pm 3.4 \mathrm{~s}$ from the beginning of the intrathecal injection and lasted $8.8 \pm 1.7 \mathrm{~s}$. Typically there were $4.0 \pm 0.6$ bursts of synchronous activity with a repetition rate of $0.427 \pm 0.14 \mathrm{~Hz}$. The mean peak frequency of discharge in each burst reached $114.8 \pm 16.3 \mathrm{~Hz}$.

BP was also increased significantly $(\mathrm{P}<0.05, \mathrm{n}=26)$ from $91.7 \pm 3.6 \mathrm{mmHg}$ to $102.4 \pm 4.3$ $\mathrm{mmHg}$. However neither heart rate $(457.9 \pm 7.8 \mathrm{bpm}$ before to $461.3 \pm 8.2 \mathrm{bpm}, \mathrm{P}>0.05)$ nor ventilation rate $(140.1 \pm 4.7$ breaths per minute before to $153.4 \pm 6.0$ breaths per minute, $\mathrm{P}>0.05$ ) were significantly affected.

\section{Spinal cord NOS inhibition with L-NAME and TRIM i.t.}

\section{Baseline effects}

Intrathecal L-NAME $(n=4)$ did not significantly affect mean arterial BP $(85.8 \pm 10.4$ $\mathrm{mmHg}$ to $94.2 \pm 10.9 \mathrm{mmHg}), \mathrm{HR}(446.2 \pm 21.2 \mathrm{bpm}$ to $433.2 \pm 20.8 \mathrm{bpm})$, the rate of ventilation (116.6 \pm 16.2 breaths per minute to $115.0 \pm 12.9$ breaths per minute) or mean firing rate within the $\operatorname{VDN}(43.8 \pm 11.5 \mathrm{~Hz}$ to $44.9 \pm 11.2 \mathrm{~Hz})$. 
Similarly, TRIM given i.t. ( $\mathrm{n}=3$ ) was without significant effect on mean arterial BP $(86.7 \pm 10.9 \mathrm{mmHg}$ to $73.1 \pm 8.2 \mathrm{mmHg}), \mathrm{HR}(508.2 \pm 31.0 \mathrm{bpm}$ to $505.8 \pm 29.8 \mathrm{bpm})$, the rate of ventilation ( $140.0 \pm 15.8$ breaths per minute to $139.0 \pm 6.7$ breaths per minute) or mean firing rate within the VDN $(64.5 \pm 15.7 \mathrm{~Hz}$ to $58.3 \pm 15.9 \mathrm{~Hz})$.

\section{On the ejaculatory response to i.v. PCA or apomorphine}

In 4 rats the intense oscillatory bursts of VDN activity, the coordinated contractions of the penile muscle and emission of seminal fluid in response to systemic administration of PCA was reversibly abolished by L-NAME i.t. (Fig 5). The mean data for the VDN response is illustrated in the bar graphs of Figure 6A-F. In a further 3 rats the specific nNOS inhibitor TRIM i.t. similarly abolished the VDN response to PCA i.v. (Fig 6 A-F). The effect of L-NAME i.t. on the response evoked by apomorphine was examined in an additional 3 rats. In 2 of these the VDN response was abolished together with other signs of ejaculation. In one rat VDN responses to i.v. apomorphine were still present but much reduced in number from 7 responses before L-NAME to 4 responses after L-NAME. These latter effects were mirrored by changes in bursts of penile muscle activity.

Insert figures 5 and 6 near this position

\section{L-NAME +L-Arginine administration i.t.}

\section{On reversal of NOS inhibition with L-Arginine}

In a separate groups of 3 rats, L-Arginine $(100 \mathrm{mM})$ was administered together with LNAME $(200 \mathrm{mM})$, intrathecally $(20-\mu \mathrm{l}) 5 \mathrm{mins}$ prior to PCA i.v. This procedure had no significant cardio-respiratory effects (data not shown). However the L-NAME inhibition of the VDN response to PCA was absent. A typical example from one rat is illustrated in Figure 7A-B. Values for all parameters measured for the response in VDN elicited by PCA i.v. were not significantly different before to those elicited by PCA i.v. alone and after L-NAME plus L-Arginine i.t. (Table 2).

Insert figure 7 and table 2 near this position

\section{Effects of L-Arginine i.t.}

In a further 5 rats the effect of L-Arginine (i.t., $100 \mathrm{mM}, 20-\mu \mathrm{l}$ ) on the PCA response was tested alone. Although there were no significant effects on blood pressure, heart rate or ventilation or the basal firing rate of activity in the VDN (data not shown), certain aspects of the response to i.v. PCA were significantly enhanced. Thus the number of synchronous 
bursts within a response was significantly increased $(6.2 \pm 1.3$ to $11.0 \pm 1.5, \mathrm{P}<0.05$, Fig $8 \mathrm{D})$ so that the individual response duration significantly increased (15.9 $\pm 3.6 \mathrm{~s}$ to $29.3 \pm 4.9 \mathrm{~s}, \mathrm{P}<0.05$, Fig $8 \mathrm{E}$ ) and there was a trend for the latency to the first response to be

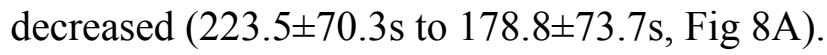

Insert figure 8 near this position

\section{Spinal cord NO donation}

\section{Baseline effects}

Intrathecal application of the NO donor sodium nitroprusside (SNP) was tested in 8 rats. This caused a significant decrease in mean arterial BP $(93.8 \pm 4.8 \mathrm{mmHg}$ to $72.3 \pm 2.6$ $\mathrm{mmHg}, \mathrm{P}<0.001)$ whilst not significantly altering heart rate $(508.9 \pm 8.4 \mathrm{bpm}$ to $514.2 \pm 9.1$ bpm, $\mathrm{P}>0.05)$ or the rate of ventilation $(137.4 \pm 5.7$ breaths per minute to $136.1 \pm 5.4$ breaths per minute, $\mathrm{P}>0.05$ ).

In two out of eight animals, SNP i.t. (1mM, 20- $\mu \mathrm{l})$ was followed by an increase in basal discharge in the VDN from $71.0 \mathrm{~Hz}$ to $76.5 \mathrm{~Hz}$ and $15 \mathrm{~Hz}$ to $23.0 \mathrm{~Hz}$ leading to a single series of oscillatory bursts in VDN (response) with a peak discharge rate of $171.0 \mathrm{~Hz}$ and $105 \mathrm{~Hz}$. This response occurred after a latency of $28.5 \mathrm{~s}$ and $24.3 \mathrm{~s}$ and lasted for $12.0 \mathrm{~s}$ and $9.1 \mathrm{~s}$. There were 6 and 4 bursts of synchronous activity within the responses, repeated at a rate of $0.510 \mathrm{~Hz}$ and $0.429 \mathrm{~Hz}$. In the remaining 6 rats, SNP i.t. increased the level of activity in the VDN from a mean of $43.3 \pm 5.0 \mathrm{~Hz}$ to $50.9 \pm 5.3 \mathrm{~Hz}(\mathrm{P}>0.05)$ but this did not result in a period of bursting activity.

\section{On the ejaculatory response to i.v. apomorphine and PCA}

In 6 rats, after testing the effect of either apomorphine ( 2 rats) or PCA (4 rats) i.v. in the induction of a response within the VDN, the effect of SNP (i.t., $1 \mathrm{mM}, 20-\mu 1$ ) was tested alone. Next SNP i.t. was applied 5 mins before intravenous apomorphine or PCA. NO donation i.t. greatly enhanced the number of oscillatory bursts within a response initiated by apomorphine i.v. (4.8 to 11.6 and 4.5 to 5.8 , apomorphine alone SNP+apomorphine respectively) in the two rats on which it was tested (Fig 9A-B). Other parameters of the apomorphine induced VDN response were not consistently altered (latency, $49.5 \mathrm{~s}$ to $137.0 \mathrm{~s}$ and $333.8 \mathrm{~s}$ to $77.0 \mathrm{~s}$; duration of response period, $2449.0 \mathrm{~s}$ to $1511.9 \mathrm{~s}$ and 630.0 $\mathrm{s}$ to $631.9 \mathrm{~s}$; number of responses, 11 to 9 and 2 to 4 ; repetition rate of bursts, $0.494 \mathrm{~Hz}$ to 
$0.300 \mathrm{~Hz}$ and $0.530 \mathrm{~Hz}$ to $0.500 \mathrm{~Hz}$; peak discharge rate of bursts, $127.8 \mathrm{~Hz}$ to $77.2 \mathrm{~Hz}$ and $179.5 \mathrm{~Hz}$ to $164.8 \mathrm{~Hz}$ ).

Similarly the VDN responses induced by PCA appeared to be facilitated by i.t. SNP. This was clearly evidenced in 1 rat (Fig 9C-D) where there was a dramatic increase in the number of oscillatory bursts and length of response. In the three other rats that failed to respond to repeated administrations of PCA i.v. $(1 \mathrm{mg} / \mathrm{kg})$, prior intrathecal application of SNP resulted in a return of ejaculatory responses in the VDN following i.v. PCA.

Insert figure 9 near this position

\section{Discussion}

We have previously described a characteristic series of responses in the nerve supplying the vas deferens that are associated with ejaculation evoked in the anaesthetised male rat by p-Chloroamphetamine (PCA) (Stafford et al, 2006a) or apomorphine (Stafford and Coote, 2006) when given intravenously. Using this model the present study provides evidence that neuronal derived nitric oxide in the lumbar spinal cord plays a significant role in the induction of a synchronised bursting pattern of sympathetic and somato-motor activity that may be representative of the neural events accompanying sexual climax. The conclusion is based on the demonstration that inhibitors of nitric oxide synthase applied to the lumbar spinal cord prevent the ejaculatory like response. Secondly this blockade can be partially overcome by the NO substrate L-Arginine. Thirdly, NO donation with sodium nitroprusside at the lumbar level enhanced certain aspects of the effect of PCA or apomorphine. Fourthly, many NOS positive neurones are located in areas of the lumbar spinal cord and sacral cord that may relay signals related to ejaculation.

The immunohistochemical study using an antibody specific against neuronal NOS revealed that as well as dense clusters of neurones in the IML at $\mathrm{L}_{1}-\mathrm{L}_{2}$, there were many NOS positive neurones located in the dorsal gray commissure above the central canal (Dun et al, 1993). The neurones have a topography and morphology similar to the sympathetic preganglionic neurones described to project in the hypogastric nerve and comprising the dorsal central autonomic nucleus that is a special feature at L1-L2 level of the spinal cord at least in the rat and guinea pig (Hancock and Peveto, 1979; Neuhuber, 1982; Barber et al, 1984; McLachlan, 1985; Nadelhaft and McKenna, 1987; Baron and Janig, 1991). However, since we did not positively identify these neurons as sympathetic 
preganglionic neurones by retrograde labelling, their identity is still open to question. A further distinct group of NOS positive neurones is apparent at the L3-L4 level, in Lamina $\mathrm{X}$ on either side and ventral to the central canal. This latter group also appears to have been labelled in the histochemical studies of Valtschanoff et al (1992) and the immunohistochemical studies of Dun et al (1993) although no attention was drawn to it. The location overlaps with that of interneurones expressing c-fos activated during the urethrogenital reflex (Marson and Gravitt, 2004). Transneuronal virus labelling of the penile muscles ischiocavernosus and bulbospongiosus or the prostate also results in the infection of interneurones in the same region of the lumbar spinal cord (Marson and McKenna, 1996; Xu et al., 2006). Furthermore neurones in this area comprise a key part of a spinal ejaculation generator (Truitt and Coolen, 2002; Carro-Juarez et al., 2003; Coolen et al., 2004). Thus there is a persuasive correlation in the location of NOS positive neurones and those neurones participating in emission and expulsion of sperm, suggesting that NO could play a key role in sexual responses.

We tested this possibility by examining the pattern of bursting nerve activity in he VDN elicited following intravenous PCA or apomorphine. These compounds were chosen because each acts by a different pathway. PCA elicits the response via a $5-\mathrm{HT}_{2 \mathrm{C}}$ receptor located, at least in part, on neurones in the spinal cord (Stafford et al., 2006b). In contrast the VDN response to apomorphine is mediated via D2/D3 receptor on neurones in supraspinal regions (Stafford et al., 2006b; Clement et al., 2007). The latter induces the effects in the spinal circuits independently of spinal 5-HT receptors since the action of apomorphine is unaffected by pre-administration of a highly selective $5-\mathrm{HT}_{2 \mathrm{C}}$ receptor antagonist (Stafford and Coote, 2006) which blocks the PCA induced responses.

A problem with the i.v administration of PCA or apomorphine is that they produce cardiovascular and respiratory effects that may secondarily induce the VDN and penile muscle responses. By measuring these effects in this study we are able to rule this out because the cardio-respiratory changes were opposite for each drug and the occurrence was not positively correlated in time or direction with the appearance of the sequence of ejaculatory responses.

An important feature of the action of PCA and apomorphine is that the VDN responses are generally very robust to repeated application. Using the third intravenous dose of 
PCA, which was $100 \%$ effective in the 6 control rats, we were able to rule out chance as a factor in the changes induced by intrathecally applied compounds. However, the lack of the clear pattern of VDN burst activity following a first dose of PCA in over $70 \%$ of animals was surprising. We had not observed this in our previous studies and have no convincing explanation, although in the present experiments unlike previous studies, all rats had undergone placement of an intrathecal catheter over the lumbar cord.

Having established a statistically valid protocol, the results convincingly show that NO is critical to the initiation of the genital response in these anaesthetised rats. Thus the intrathecal application of either the NOS inhibitor L-NAME or the more selective nNOS inhibitor TRIM abolished the series of responses associated with emission and ejaculation evoked by i.v. PCA as well as those by apomorphine. That this is an effect on the NO pathway is strengthened by the action of the NO substrate L-Arginine in reversing the actions of L-NAME. This is further supported by the action of intrathecal donation of NO by SNP, which given prior to i.v. PCA or apomorphine, potentiated the burst responses in the VDN, as measured by increases in the number of oscillatory bursts and duration of response. Of particular importance was that intrathecal SNP on its own was able to induce an oscillatory pattern of responses in 2 rats and it also appeared to enhance responses to PCA in another 3 rats.

The site of action of $\mathrm{NO}$ within the lumbar spinal cord was not specifically addressed in this study. However several pieces of the evidence may help identify how and where NO is acting to induce the series of observed motor responses linked with sexual behaviour. Potential sites are the IML and dorsal central autonomic nuclei in the $\mathrm{L}_{1}-\mathrm{L}_{2}$ spinal cord where as we confirmed in the present study many neurones express NOS (Valtschanoff et al., 1992; Dun et al., 1993). As already suggested those in the dorsal central autonomic nuclei may be sympathetic preganglionic neurones projecting to the VDN via the hypogastric nerve. Therefore one mechanism maybe NO formation and release in these neurones as a result of $\mathrm{Ca} 2+$ influx which has been shown to enhance excitatory post synaptic potential in sympathetic preganglionic neurones in rat spinal cord slice in vitro (Wu and Dunn, 1995). This probably naturally occurs via presynaptic potentiation of neurotransmitter release and so we might postulate a similar action at terminals on sympathetic preganglionic neurones, of pathways activated by either PCA or 
apomorphine in the present study. However this may not be the main contributor because the increase in VDN firing discharge rate, typically following PCA, appeared to be unaffected by NOS inhibition. The data are more consistent with an NO involvement at a pre-sympathetic site such as an ejaculatory pattern generator like that described to lie in lamina $\mathrm{X}$ at the $\mathrm{L}_{3}-\mathrm{L}_{4}$ level (Truitt and Coolen, 2002; Carro-Juarez et al., 2003; Coolen et al., 2004), where NOS positive neurones were observed in the present study. Any action of NOS inhibitors at this latter site would more easily explain their effects in preventing not only the VDN response but also the rhythmic contraction of the penile muscle. Similarly the induction of the entire ejaculatory response by L-Glutamic acid and SNP suggest a site common to both autonomic and somatic motor outflows. In this regard we found a conspicuous absence of NOS immunoreactive neurones in the ventral horn of the lumbar cord at locations where bulbospongiosus or ischiocavernosus muscle motoneurones have been identified by retrograde labelling (Scroder, 1980; Marson and McKenna, 1996; Tang et al., 1999). Therefore the inhibitory effect of L-NAME or TRIM could not have occurred on these neurones. We are aware that this study only examined the role of NO in those pathways mediating the action of apomorphine or PCA.

The same may not be the case for reflexly activated ejaculation that appears to depend on rhythmic pudendal nerve afferent input at the sacral and upper lumbar segments of the spinal cord (Marson and Gravitt, 2004). This might also implicate either neurones in the superficial laminae of the dorsal horn or groups of interneurones lying in lamina $\mathrm{X}$ between $\mathrm{L}_{5}-\mathrm{S}_{1}$ (McKenna and Nadelhaft, 1986; Marson and Gravitt, 2004; Xu et al., 2006) amongst which we observed nNOS positive neurones. However to generate a coordinated activation of the diverse outputs there needs to be a means of synchronisation. We speculate that his is best understood in terms of a single generator.

Thus an action of NO primarily at an ejaculation generator would mean an enhancement of the ejaculatory response to all afferent stimuli. Therefore we suggest that reflex activation of ejaculation by signals from the nerves of the penis would be similarly facilitated, to the PCA or apormorphine responses, but this important question remains to be answered. 


\section{Conclusion}

In conclusion this study in the anaesthetised rat has shown that the rhythmic responses in the sympathetic nerve to the vas deferens and of the simultaneous coordinated contraction of penile muscles, comprising the ejaculatory response to i.v. PCA and apomorphine, involves NO formation and release in the lumbosacral spinal cord. The sympathetic preganglionic neurones supplying the vas deferens, parasympathetic neurones, somatosensory neurones in the lumbosacral spinal cord as well as neurones of an ejaculator pattern generator are all possible sites for the action of NO. The extent of the NO influence suggests it is pivotal for generation of rhythmic responses in autonomic and somatic outflows concerned with ejaculation in the anaesthetised preparation and may well be so in conscious animals.

\section{Acknowledgments}

This work was funded by a donation from Plethora Solutions Ltd. 


\section{References}

Anderson CR, 1992. NAPDH diaphorase-positive neurones in the rat spinal cord include a subpopulation of autonomic preganglionic neurones. Neurosci Lett., 139, 280-284.

Anderson CR, McAllen RM and Edwards SL, 1995. Nitric oxide synthase and chemical coding in cat sympathetic postganglionic neurones. Neurosc, 68; 255-264.

Barber RP, Phelps PE, Houser CR, Crawford GD, Salvaterra PM and Vaughn JE, 1984. The morphology and distribution of neurones containing choline acetlytransferase in the adult rat spinal cord: an immunocytochemical study. J Comp Neurol, 229, 329-346.

Baron R and Janig W, 1991. Afferent and sympathetic neurones projecting into lumbar visceral nerves of the male rat. J Comp Neurol; 314: 429-36.

Blottner D and Baumgarten HG, 1992. Nitric oxide synthase (NOS)-containing sympathoadrenal cholinergic neurons of the rat IML-cell column: evidence from histochemistry, immunohistochemistry, and retrograde labelling. J Comp Neurol, $316(1): 45-55$.

Carro-Juarez M, Cruz SL, Rodriguez-Manzo G, 2003. Evidence for the involvement of a spinal pattern generator in the control of the genital motor pattern of ejaculation. Brain Res; 975: 222-28.

Clement P, Bernabe J, Denys P, Alexandre L and Giuliano F, 2007. Ejaculation induced by i.c.v. injection of the preferential dopamine D3 receptor agonist 7-hydroxy-2-(DI-Npropylamino)tetralin in anaesthetised rats. Neurosci; 145: 605-610.

Coolen LM, Allard J, Truitt WA and McKenna KE, 2004. Control and regulation of ejaculation. Physiology and behaviour; 83: 203-215. 
Dun NJ, Dun SL, Wu SY, Forstermann U, Schmidt HH and Tseng LF, 1993. Nitric oxide synthase immunoreactivity in the rat, mouse, cat and squirrel monkey spinal cord. Neurosc, 54; 845-857.

Garcia M, Celuch SM and Adler-Graschinsky E, 1997. Possible participation of spinal cord nitric oxide in the control of blood pressure in anaesthetised rats. Brain Res, 764; $67-74$.

Hakim MA, Hirooka Y, Coleman MJ, Bennett MR, Dampney RA, 1995. Evidence for a critical role of nitric oxide in the tonic excitation of rabbit renal sympathetic preganglionic neurones. $J$ Physiol; 482: 401-407.

Hancock MB and Peveto CA, 1979. A preganglionic autonomic nucleus in the dorsal gray commissure of the lumbar spinal cord of the rat. J Comp Neurol; 183: 65-72.

Lee SB, Koh H, Kim ON, Sung KW, and Kim SY, 1996. Intrathecal administration of sodium nitroprusside, a nitric oxide donor, increases blood pressure in anaesthetised rats. Neurosci Lett; 203: 53-56.

Marson L and Gravitt K, 2004. Spinal neurones activated with the urethogenital reflex in the male rat. Brain Res; 1026: 108-15.

Marson L and McKenna KE, 1996. CNS cell groups involved in the control of the ischio cavernosus and bulbospongiosus muscles: a transneuronal tracing study using pseudorabies virus. J Comp Neurol; 374: 161-79.

McLachlan EM, 1985. The components of the hypogastric nerve in male and female guinea pigs. J Auton Nerv Syst; 13: 327-42.

Nadelhaft I and McKenna KE, 1987. Sexual dimorphism in sympathetic preganglionic neurones of the rat hypogastric nerve. J Comp Neurol; 256: 308-15. 
Neuhuber W, 1982. The central projections of viscera primary afferent neurones of the inferior mesenteric plexus and hypogastric nerve and the location of the related sensory and preganglionic cell bodies in the rat. Anat Embryol; 164: 413-25.

Saito S, Kidd GJ, Trapp BD, Dawson TM, Bredt DS, Wilson DA, Traystman RJ, Snyder SH, Hanley DF, 1994. Rat spinal cord neurones contain nitric oxide synthase. Neurosci; 59: 447-456.

Schroder HDAA, 1980. Organisation of the motor neurones innervating the pelvic muscles of the male rat. J Comp Neurol; 192: 567-587.

Stafford SA, and Coote JH, 2006. Activation of D2-like receptors induces sympathetic climactic-like responses in male and female anaestetised rats. Br J Pharmacol. 148: 51016.

Stafford SA, Bowery NG, Tang $\mathrm{K}$ and Coote JH, 2006a. Activation by pChloroamphetamine of the spinal ejaculatory pattern generator in anaesthetised male rats. Neurosci. 140(3); 1031-1040.

Stafford SA, Tang K and Coote JH, 2006b. Activation of lumbosacral 5- $\mathrm{HT}_{2 \mathrm{C}}$ receptors induces bursts of rhythmic activity in sympathetic nerves to the vas deferens in male rats. Br J Pharmacol. 148(8): 1083-90.

Storkson VR, Kjorsvik A, Tjolsen A and Hole K, 1996. Lumbar catheterisation of the spinal subarachnoid space in the rat. J Neurosci Methods. 65: 167-72.

Tang Y, Ramph D, Giuliano F and Ugolini G, 1999. Spinal and brain circuits to interneurones of the bulbospongiosus muscle: retrograde transneuronal tracing with rabies virus. J Comp Neurol; 414, 167-92. 
Truitt WA and Coolen LM, 2002. Identification of a potential ejaculation generator in the spinal cord. Science; 297: 1566-69.

Valtschanoff JG, Weinberg RJ and Rustioni A, 1992. NADPH diaphorase in the spinal cord of rats. J Comp Neurol, 321(2):209-22.

Wu SY and Dun NJ, 1995. Calcium-activated release of nitric oxide potentiates excitatory synaptic potentials in immature rat sympathetic preganglionic neurons. $J$ Neurophysiol, 74(6):2600-3.

Wu SY and Dun NJ, 1996. Potentiation of IPSCs by nitric oxide in immature rat sympathetic preganglionic neurones in vitro. J Physiol, 495(2):479-90.

Xu C, Giuliano F, Yaici ED, Conrath M, Trassard O, Benot G and Verge D, 2006. Identification of lumbar spinal neurones controlling simultaneously the prostate and the bulbospongiosus muscles in the rat. Neurosci; 138: 561-73.

Yang A, Smith L and Coote JH, 2004. Paraventricular nucleus activation of renal sympathetic neurones is synaptically depressed by nitric oxide and glycine acting at a spinal level. Neurosci, 124(2): 421-8.

Zheng H, Mayhan WG, Bidasee KR and Patel KP, 2006. Blunted nitric oxide-mediated inhibition of sympathetic nerve activity within the paraventricular nucleus in diabetic rats. Am J Physiol, 290, R992-R1002. 

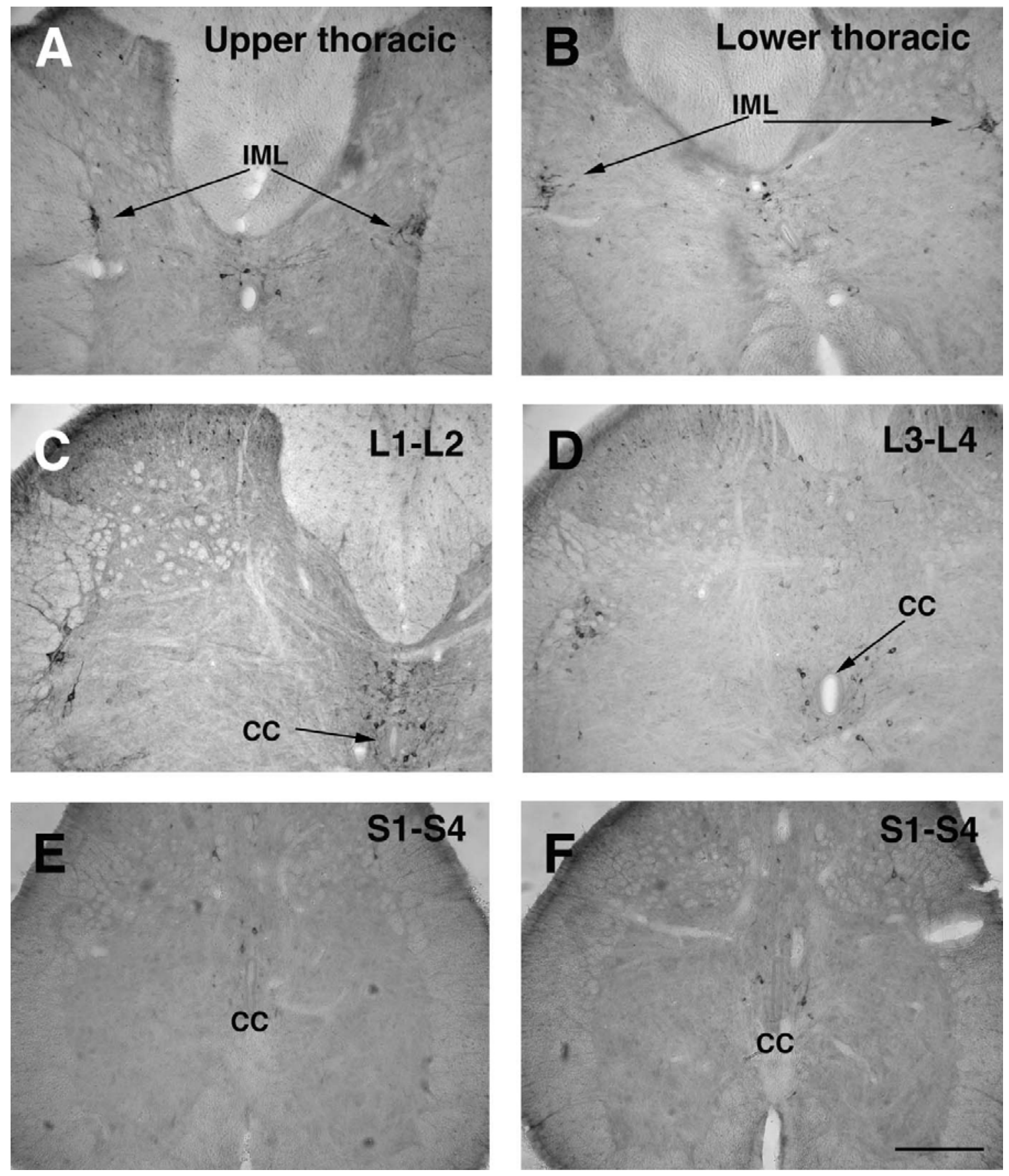

\section{Figure 1}

Transverse sections of rat spinal cord showing the distribution of neurones stained for nNOS. A, B: Thoracic segments, nNOS positive neurones are present in the principal sympathetic nucleus (IML) and in the central autonomic area (CA). C, D: Lumbar segments, nNOS stained neurons are distributed in the IML and CA above CC and ventrally around the CC. E, F: Sacral segments, a few nNOS positive neurones are present above the CC. Bar $=160 \mu \mathrm{m}$. Abbrev. IML-intermediolateral cell column; CCcentral canal. 

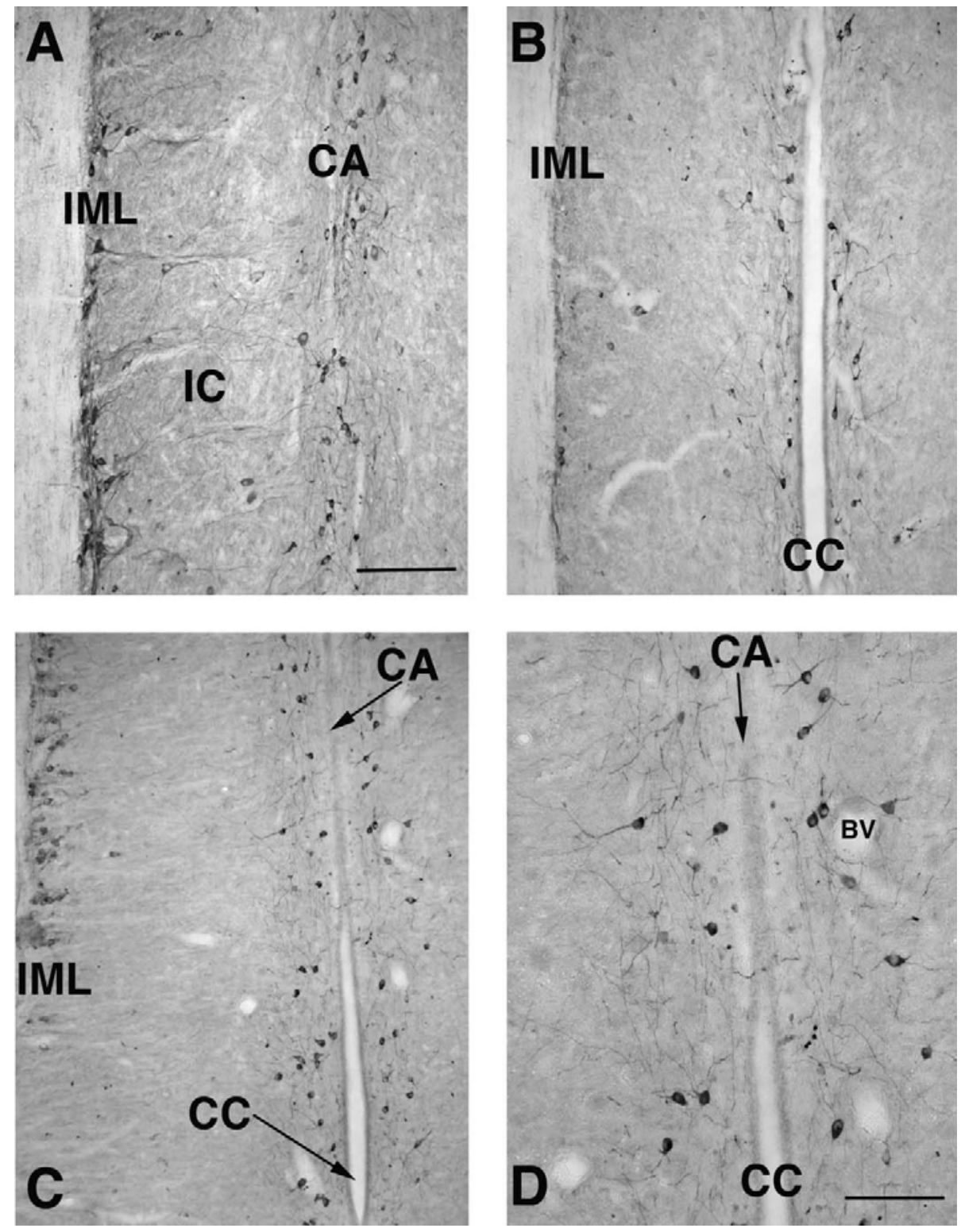

\section{Figure 2}

Distribution of nNOS positive neurones in horizontal section of rat spinal cord (T8-L4). A, B: In lower thoracic segments nNOS positive neurones were found within the main sympathetic nuclei (IML, IC, CA) and either side of the central canal (CC). C: In lumbar segments nNOS staining was found in the IML, CA and extending ventrally around CC. D: Higher magnification of $\mathrm{C}$. nNOS positive neurones with extensive dendritic arborisation were sited close to CA and more ventrally surrounding the CC. Note the close association of some nNOS positive cell bodies and dendrites with blood vessels. Abbreviations BV- blood vessel, IML -intermediolateral cell column; IC-intercalated region CA- central autonomic area; CC-central canal. A-C Bar $=160 \mu \mathrm{m}$; D Bar $=80$ $\mu \mathrm{m}$. 


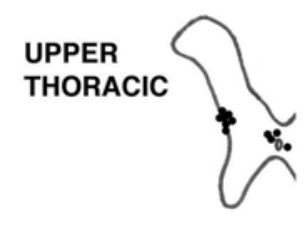

L1

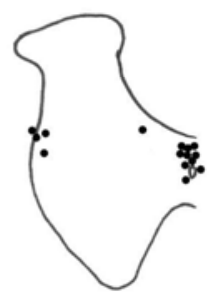

L3

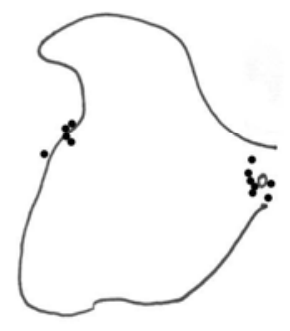

S1

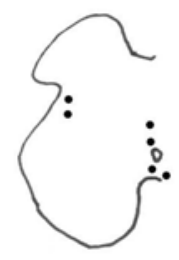

S3

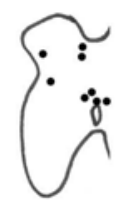

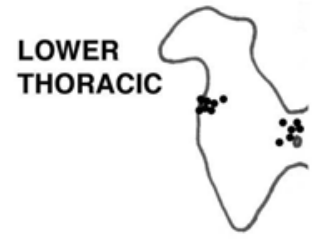

L2

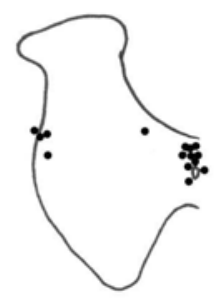

L4

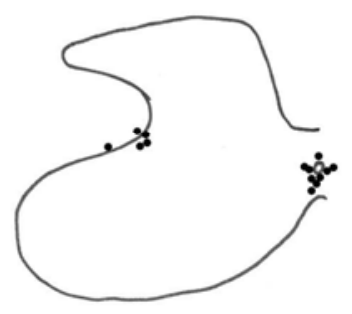

S2

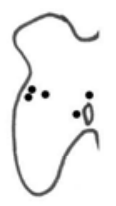

S4

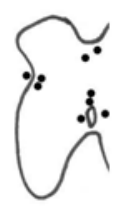

\section{Figure 3.}

Schematic of the gray matter on the left side at representative levels of the rat spinal cord showing the distribution of nNOS positive neurones in the principle autonomic nuclei, ventral horn, intermediate gray and central gray area: mapping from one rat. Each dot represents one cell body. 


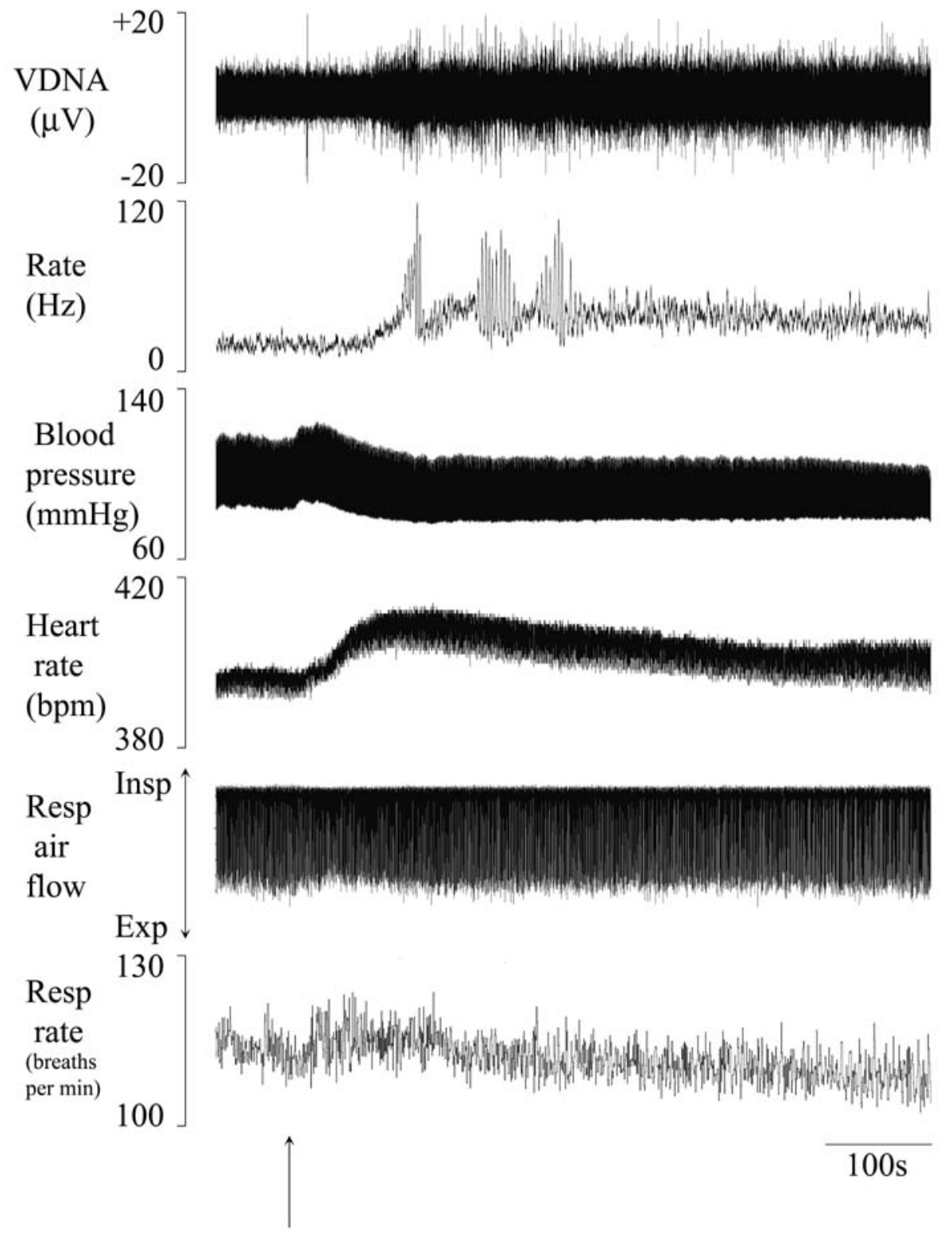

\section{Figure 4.}

Effect of $1 \mathrm{mg} / \mathrm{kg}$ PCA (arrow, i.v.) on raw vas deferens nerve activity (VDNA), integrated firing rate within the vas deferens nerve, blood pressure, heart rate, respiratory airflow movements and rate of respiration from a typical experiment. 


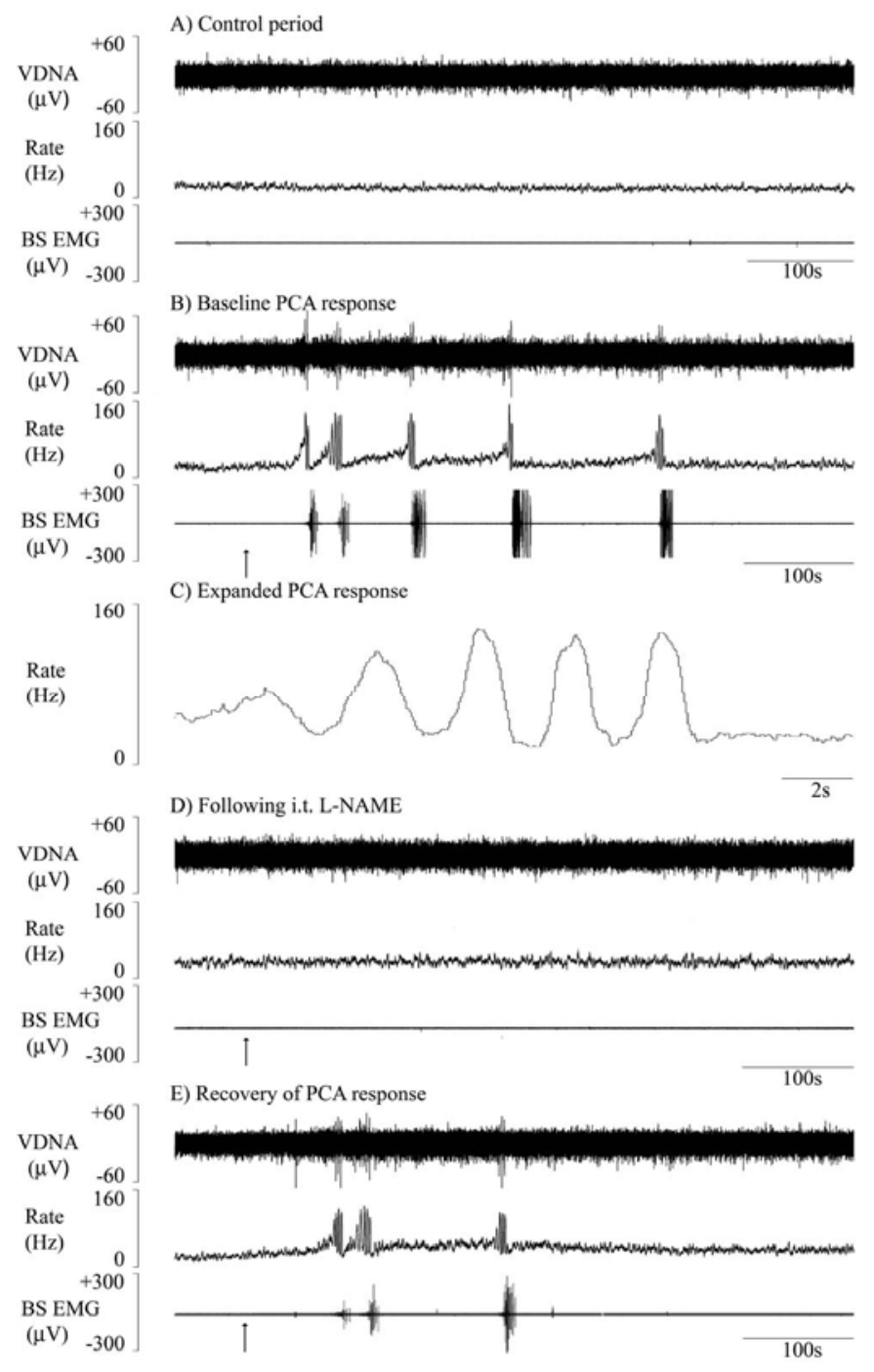

\section{Figure 5.}

PCA induced bursting activity of the VDN and EMG of the bulbospongiosus muscle (BS EMG) and its abolition by intrathecal application of $\mathrm{N}^{(\mathrm{G})}$ nitro-L-Arginine methyl ester (L-NAME). A. A control period of raw VDN activity, the integrated rate meter record and BS EMG. B. Period of synchronous bursting within the VDN and BS EMG after 1 $\mathrm{mg} / \mathrm{kg}$ PCA (arrow, i.v.). C. An expanded trace of the integrate rate meter record of VDN activity, 70s after administration of $1 \mathrm{mg} / \mathrm{kg}$ PCA. D. The neural and EMG response after $1 \mathrm{mg} / \mathrm{kg}$ PCA (i.v., arrow) following intrathecal application of L-NAME at $\mathrm{L}_{2}(200 \mathrm{mM}$, $20 \mu \mathrm{l})$. E. Recovery of the responses to i.v. PCA (1mg/kg, arrow) 60 minutes after LNAME application. 

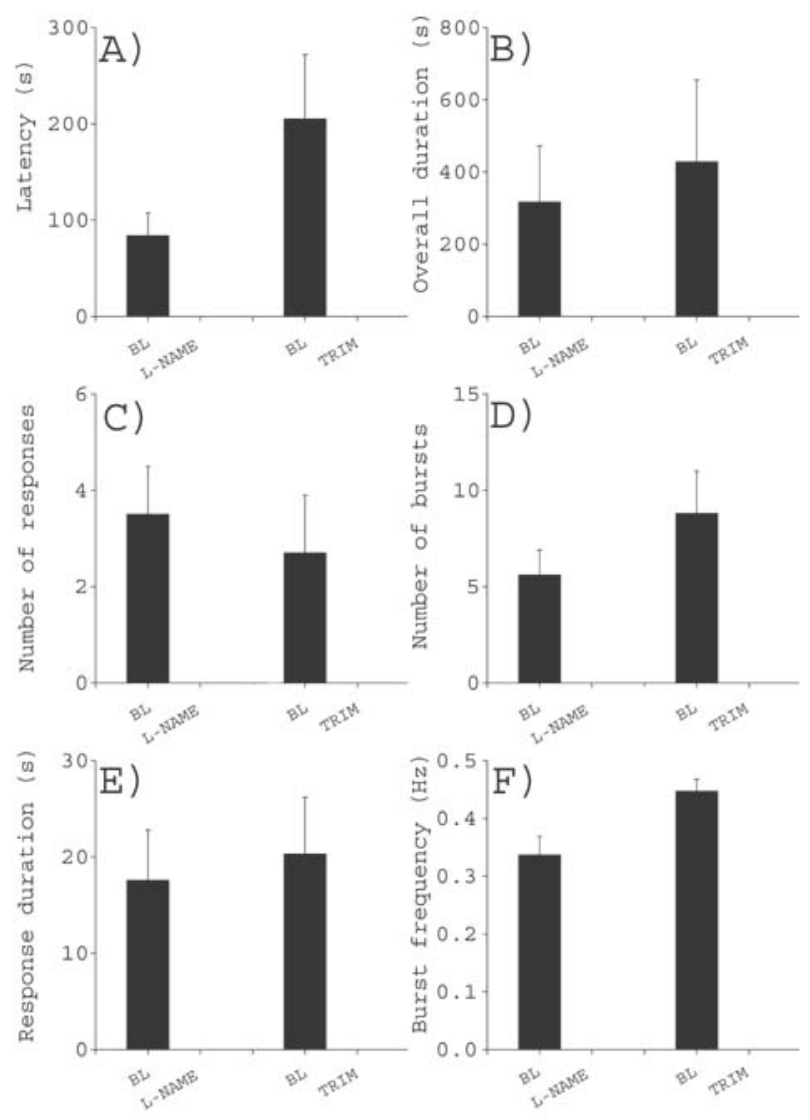

Figure 6.

Summary of the effect of NOS inhibition using the non specific NOS inhibitor $\mathrm{N}^{(\mathrm{G})}$ nitroL-Arginine methyl ester (L-NAME) and the neuronal specific NOS inhibitor 1-(2trifluoro-methylphenyl) imidazole (TRIM) on the VDN response to $1 \mathrm{mg} / \mathrm{kg}$ PCA (i.v.). A) latency to first response, B) overall duration of response(s), C) number of responses, D) number of synchronous oscillatory bursts within each response, E) individual response duration, D) frequency of synchronous oscillatory bursts. BL: baseline PCA response prior to L-NAME or TRIM application. 


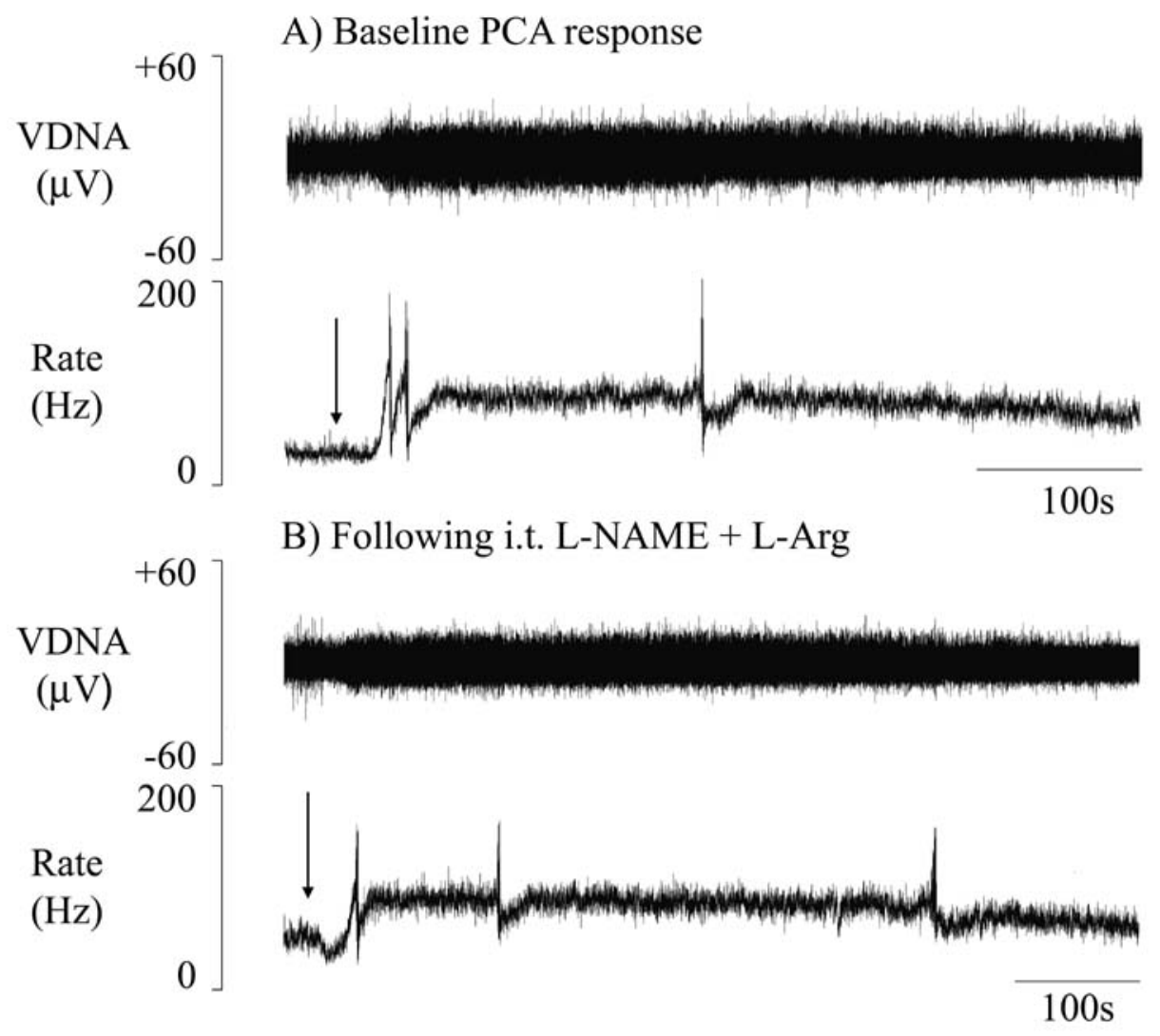

\section{Figure 7.}

PCA induced bursting activity of the VDN and reversal of the abolition of the response following intrathecal administration of $\mathrm{N}^{(\mathrm{G})}$ nitro-L-Arginine methyl ester (L-NAME) with the simultaneous application of L-Arginine (L-NAME+L-Arg). A. Baseline response to $1 \mathrm{mg} / \mathrm{kg}$ PCA (i.v., arrow). B. Response to i.v. (arrow) PCA following intrathecal application of L-NAME $(200 \mathrm{mM})$ and L-Arg $(200 \mathrm{mM})$ at $\mathrm{L}_{2}(20 \mu \mathrm{l})$. 

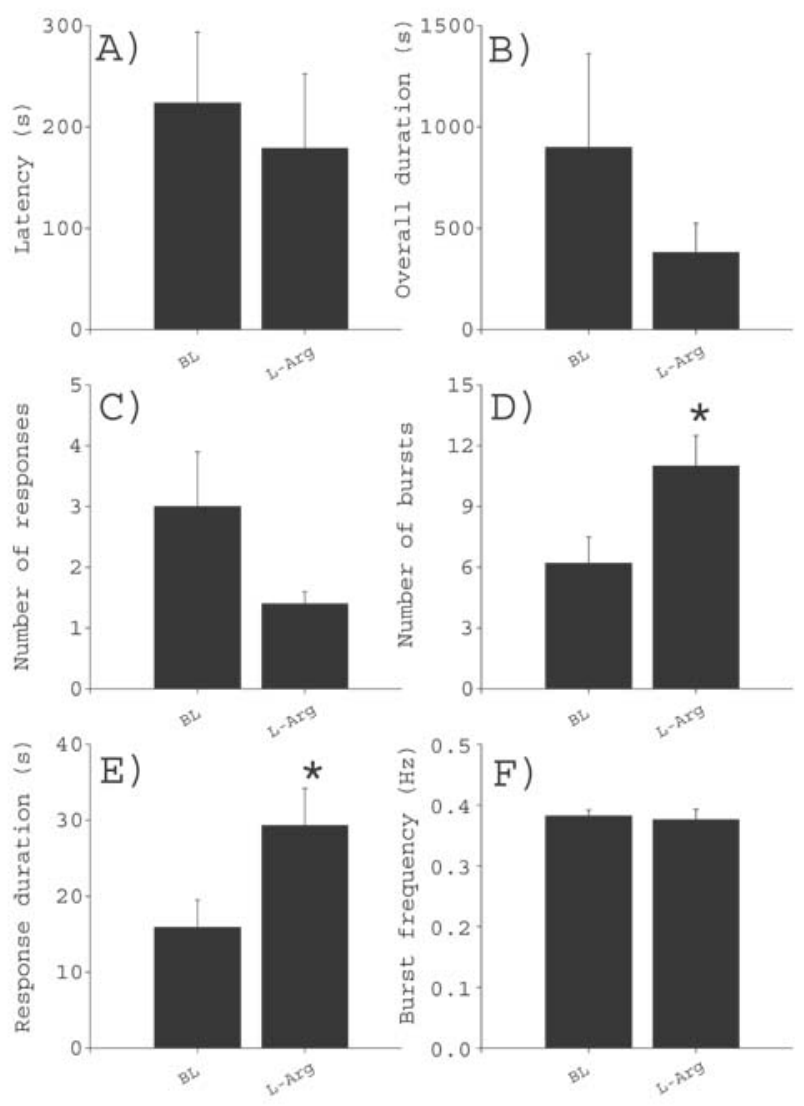

Figure 8.

Summary of the effect of intrathecal application of L-Arginine (L-Arg, 200mM, $20 \mu$ on the VDN response to $1 \mathrm{mg} / \mathrm{kg}$ PCA (i.v.). A) latency to first response, B) overall duration of response(s), C) number of responses, D) number of synchronous oscillatory bursts within each response, E) individual response duration, D) frequency of synchronous oscillatory bursts. BL: baseline PCA response prior to L-Arg application. ${ }^{*} \mathrm{P}<0.05$, paired students t-test. 
A) Baseline Apomorphime

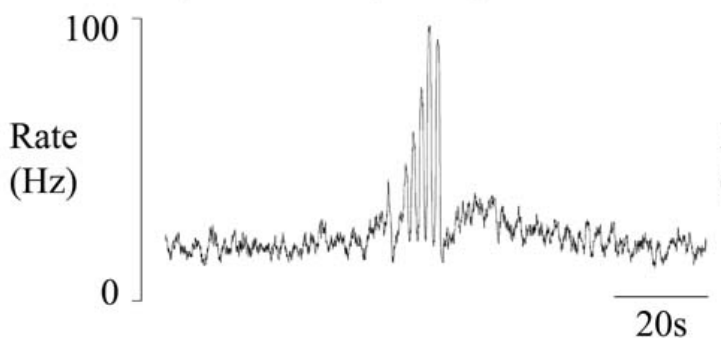

C) Baseline PCA

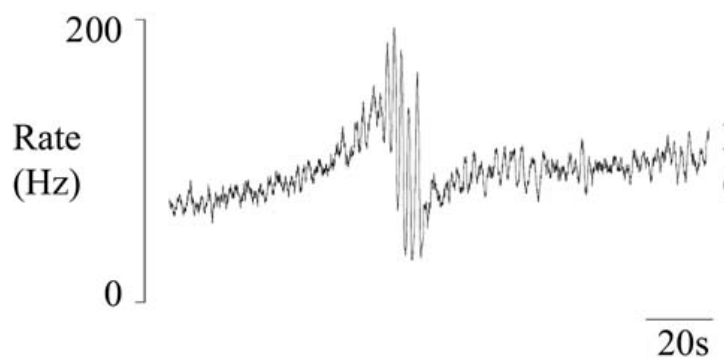

B) Apomorphine following i.t. SNP

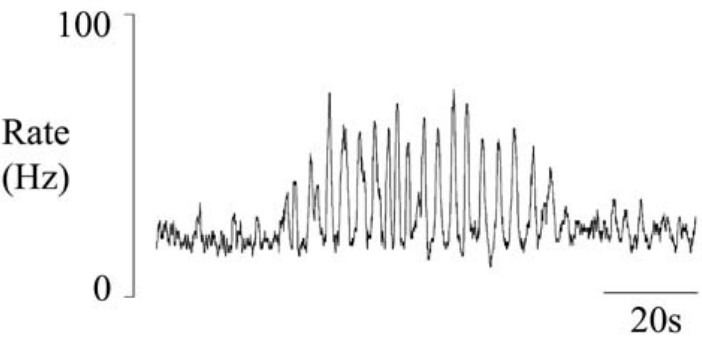

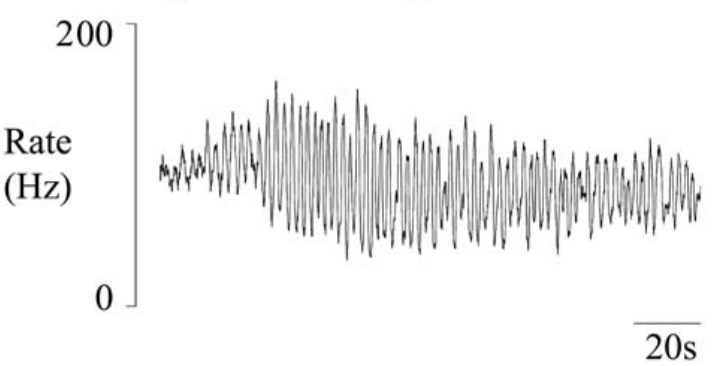

\section{Figure 9.}

Enhancement of the response to $1 \mathrm{mg} / \mathrm{kg}$ apomorphine (i.v.) and $1 \mathrm{mg} / \mathrm{kg}$ PCA following intrathecal application of SNP $(1 \mathrm{mM}, 20 \mu \mathrm{l})$. Examples of a baseline response within the VDN after apomorphine (A) and PCA (C) and again following SNP application (B: apomorphine, D: PCA). 